\title{
Carbon Dioxide Discharge Test Modelling
}

ROBERT ZALOSH and CHENG WAI HUNG

Center for Firesafety Studies

Worcester Polytechnic Institute

Worcester, Massachusetts 01609

\section{ABSTRACT}

A mathematical model is formulated here to calculate the time dependent pressures, temperatures, and gas concentrations developed during and following the discharge of carbon dioxide into an enclosure with a known leakage area. The model is based on conservation of mass, energy, and carbon dioxide with assumed spatial distributions of temperature and carbon dioxide concentration. The formation and subsequent evaporation of solid phase carbon dioxide, and the cooling of the enclosure walls and floor are determined as part of the solution.

Model calculations simulating a series of discharge tests previously conducted in a $993 \mathrm{~m}^{3}$ enclosure are compared to the reported test data. Results show good agreement with concentration data at all times and elevations in the enclosure. Pressures and temperatures show good agreement during the discharge, and somewhat overestimate pressure and temperature recovery following discharge.

KEYWWORDS: carbon dioxide, dischargetest, mathematical model.

\section{INTRODUCTION}

Restrictions on the use of Halons have motivated renewed interest in carbon dioxide total flooding extinguishing systems. An important consideration in the approval of carbon dioxide total flooding systems is the successful completion of a full discharge test to verify, as per NFPA 12 [1], that carbon dioxide is indeed discharged through the system piping, and that the design concentration is achieved and maintained for the required hold time. Another consideration for tight enclosures is to confirm that the maximum pressure developed during discharge is below the design strength of the enclosure.

There are several disincentives to conducting carbon dioxide discharge tests. These include: 1) personnel safety concerns associated with the high $\mathrm{CO}_{2}$ concentrations and the corresponding low $\mathrm{O}_{2}$ concentrations; 2) possible cold shock damage to vulnerable electronic equipment in the discharge 
path; 3) possible pressure damage to tight enclosures with inadequate venting or leakage area; and 4) the cost and inconvenience incurred by the tests.

In view of these disincentives and the success of recent alternatives to Halon 1301 discharge testing, it is logical to explore similar alternatives for $\mathrm{CO}_{2}$ total flooding systems. The particular alternative explored in this paper is the combined use of a fan pressurization test to determine enlosure leakage area, together with a mathematical model to calculate enclosure pressures and carbon dioxide concentration build up and decay. (A separate test of system piping would also be needed.) Before describing the model developed for this purpose, a review of the relevant properties and extinguishment requirements for carbon dioxide and for Halon 1301 will help determine the applicability of typical Halon discharge test models (e.g. Ref 2) for $\mathrm{CO}_{2}$ installations.

The $\mathrm{CO}_{2}$ concentration requirements for extinguishment (34- $75 \mathrm{v} \%$ ) are an order-of-magnitude larger than the corresponding Halon 1301 requirements ( $5 \mathrm{v} \%)$. Halon systems have been required to discharge and achieve design concentration within 10 seconds, while $\mathrm{CO}_{2}$ systems discharge over a considerably longer period of time. The molecular weight for Halon 1301 is 3.4 times as large as that for carbon dioxide. Therefore, gravitational settling effects (and the possible formation of two distinct layers with different densities) should be more significant for Halon 1301 than for $\mathrm{CO}_{2}$. Since the $\mathrm{CO}_{2}$ heat of vaporization /sublimation $(570 \mathrm{~kJ} / \mathrm{kg}$ ) is almost five times as large as that for Halon $1301, \mathrm{CO}_{2}$ discharge will cause substantially more cooling of the enclosure than will Halon.

Based on these property differences, we should not expect a model developed for Halon 1301 discharge to be applicable to $\mathrm{CO}_{2}$. In order to simulate $\mathrm{CO}_{2}$ discharge tests, it is necessary to use a model that accounts for simultaneous heat transfer, $\mathrm{CO}_{2}$ discharge, and leakage effects with realistic distributions of temperature and concentration in the enclosure, and with an a provision to calculate pressure changes. The model developed in [3] and summarized herein is based on this premise.

\section{MATHEMATICAL MODEL}

Since spatial distributions and dominant phenomena differ during and following $\mathrm{CO}_{2}$ discharge, separate formulations are developed for the two different stages of a discharge test.

\section{Discharge Model}

Pertinent phenomena during discharge are shown schematically in Figure 1. Carbon dioxide is injected into the enclosure in both gaseous and solid form, with the solid fraction determined by treating the discharge process as an isentropic expansion from storage temperature and pressure to atmospheric pressure. Some of the solid phase $\mathrm{CO}_{2}$ vaporizes as it settles; the rest forms an evaporating layer on the enclosure floor. Cooling of the air to vaporize the $\mathrm{CO}_{2}$ causes the enclosure temperature to rapidly drop to the $\mathrm{CO}_{2}$ sublimation point, $-79^{\circ} \mathrm{C}$. Subsequent heat transfer from the enclosure walls gradually raises the gas-air temperature.

Leakage of air into and $\mathrm{CO}_{2}$-air mixture out of the enclosure is governed by the local pressure difference across the leakage area. A hydrostatic pressure distribution is assumed. Gas concentration, temperature, and density are assumed to be uniform due to the intense mixing and turbulence associated with the discharge from the nozzles. 
Governing equations used to simulate this representation of the discharge are conservation of mass, carbon dioxide, and energy, and the associated mixture ideal gas law. Mass conservation is written as follows.

$$
\frac{d(\rho V)}{d t}=\left(1-R_{x}\right) * G-\sum \dot{m}_{i}+\dot{m}_{v 1}+\dot{m}_{v 2}
$$

where

$\mathrm{p}=$ density of $\mathrm{CO}_{2}$-air mixture,

$\mathrm{V}=$ enclosure volume,

$\dot{m}_{\mathbf{i}}=$ leakage out (in if negative) through leakage area $A_{i}$

$\dot{m}_{\mathrm{vl}}=$ rate of $\mathrm{CO}_{2}$ evaporated from falling solid particles,

$\dot{m}_{\sqrt{2}}=$ rate of $\mathrm{CO}_{2}$ evaporated from the floor layer.

$\mathrm{R}_{\mathrm{x}}=$ fraction of $\mathrm{CO}_{2}$ flashing to solid during discharge,

$\mathrm{G}=\mathrm{CO}_{2}$ mass discharge rate.

Leakage in or out of the enclosure is calculated from the following orifice equations with discharge coefficient, $\mathrm{C}_{\mathrm{d}}$

$$
\begin{array}{ll}
\dot{m}_{i}=C_{d} A_{i}\left(2 p\left(P_{i}-P_{i a}\right)\right)^{0.5} & \text { for } P_{i} \geq P_{i a} \\
\dot{m}_{i}=-C_{d} A_{i}\left(2 P_{a}\left(P_{i a}-P_{i}\right)\right)^{0.5} & \text { for } P_{i a}>P_{i}
\end{array}
$$

where

$A_{i}=$ area of ith leakage opening,

$P_{i}=$ internal pressure at elevation of ith leakage opening,

$P_{\mathrm{it}}=$ external (ambient) pressure at elevation of $A_{i}$

$\dot{m}{ }_{i}=$ mass flow rate through $A_{i}$

If the total leakage area is known but the distribution and elevations of individual areas are not known, the distribution producing the largest leakage rates is one half the area at the bottom and the other half at the top of the enclosure. This is what is used in the model, unless otherwise specified.

The evaporation of solid $\mathrm{CO}_{2}$ particles falling to the floor is calculated using the following representation of the Spalding mass transfer correlation.

$$
\dot{m}_{v 1}=\frac{h_{1}}{C_{p}} \ln (1+B)\left(\pi d^{2}\right)\left(\frac{R G t_{p}}{\frac{1}{6} \rho_{c r} \pi d^{3}}\right)
$$


where

$h_{1}$ is the heat transfer coefficient for the falling particle,

B is the Spalding B number; i.e. the ratio of sensible heat to the heat of vaporization,

$\mathrm{d}$ is particle diameter,

$\mathrm{G}$ is the mass discharge rate of $\mathrm{CO}_{2}$ into the enclosure,

$t_{p}$ is the time required for the particle to fall from the nozzle to the floor,

$\varrho_{c B}$ is density of solid $\mathrm{CO}_{2}$ particles,

and

$\mathrm{C}_{\mathrm{p}}$ is the specific heat of the $\mathrm{CO}_{2}$-air mixture.

The particle settling time, $t_{p}$, is calculated using the average of the particle terminal velocity and the discharge velocity from the nozzle. The Nusselt number used to calculate the heat transfer coefficient is determined from a forced convection correlation using a Reynolds number based on the particle terminal velocity. The full set of supporting equations is given in Reference 3 .

Using the heat and mass transfer analogy, the rate of evaporation from the layer of solid carbon dioxide deposited on the floor is:

$$
\dot{m}_{v 2}=h_{2} A\left(\frac{G t}{M}\right)\left(\rho_{\varepsilon}-\rho \frac{x M_{c}}{x M_{c}+(1-x) M_{a}}\right)
$$

where

$\mathrm{h}_{2}$ is the heat transfer coefficient at the surface of the deposited $\mathrm{CO}_{2}$ layer,

$A$ is the floor area,

$\mathrm{M}$ is the total mass of $\mathrm{CO}_{2}$ discharged,

$\rho_{c}$ is the density of gas phase $\mathrm{CO}_{2}$ at the enclosure temperature,

$\mathrm{X}$ is the volume (mole) fraction of $\mathrm{CO}_{2}$ in the gas mixture,

$\mathrm{M}_{\mathrm{c}}$ is the molecular weight of $\mathrm{CO}_{2}$ (44),

and

$\mathrm{M}_{\mathrm{a}}$ is the molecular weight of air (28.8).

The heat transfer cofficient, $h_{2}$, is determined from a turbulent flow Reynolds number correlation using the average velocity of the falling $\mathrm{CO}_{2}$ particles and the induced velocity due to the leakage flow at the bottom of the enclosure. Details are given in (3).

The equation describing conservation of carbon dioxide is

$$
\frac{d\left(\mathrm{p} y_{m} V\right)}{d t}=\left(1-R_{x}\right) G+m_{v 1}+m_{v 2}-\left.Y_{m} \sum_{m_{l}}\right|_{\left(m_{t}(0)\right.}
$$

The relationship between $\mathrm{CO}_{2}$ volume fraction, $\mathrm{x}$, and mass fraction, $\mathrm{y}_{\mathrm{m}}$, is

$$
\begin{aligned}
y_{m} & =\frac{x M_{c}}{(1-x) M_{a}+x M_{c}} \\
\text { i.e. } \quad x & =\frac{y_{m} M_{a}}{\left(1-y_{m}\right) M_{c}+y_{m} M_{e}}
\end{aligned}
$$

Substituting Eqns 1 and 7 into the expanded form of Eqn 6, we obtain for conservation of $\mathrm{CO}_{2}$ : 


$$
\rho V \frac{d y_{m}}{d t}=\left[\left(1-R_{x}\right) G+m_{v 1}+m_{v 2}\right]\left(1-y_{m}\right)-y_{m} \sum m_{t \mid m_{1} / 0}
$$

Conservation of energy for the system depicted in Fig 1 can be written verbally as

Rate of System Internal Energy Change $=$ Rate of Energy added by $\mathrm{CO}_{2}$ discharge $\left(\mathrm{E}_{1}\right)$ - Rate of Energy absorbed by evaporation $\left(\mathrm{E}_{2}\right)$ - Rate of Energy lost by leakage flow $\left(\mathrm{E}_{3}\right)+$ Rate of Heat Transferred through Enclosure Boundary $\left(\mathrm{E}_{4}\right)$

The left hand side of this equation is

$$
\frac{d\left(\rho V U+V H_{w t} W\right)}{d t}=V U \frac{d \rho}{d t}+\rho V \frac{d U}{d t}+V H_{w t} \frac{d W}{d t}+V W \frac{d H_{w t}}{d t}
$$

where

$\mathrm{U}$ is the internal energy per unit mass of $\mathrm{CO}_{2}$-air mixture, $\mathrm{W}$ is the mass of water vapor per unit enclosure volume,

and $\mathrm{H}_{\mathrm{w}}$ is water vapor heat of condensation.

The last two terms in Eqn 9 can be rewritten in terms of water vapor specific heat, $\mathrm{C}_{\mathrm{pww}}$ liquid water specific heat, $\mathrm{C}_{\mathrm{pw}}$ temperatures, and the saturated water vapor concentration, $\mathrm{W}_{\mathrm{s}}$, at temperature $\mathrm{T}$.

On the right hand side of the energy equation, the first three terms are

$$
\begin{aligned}
& E_{1}=\left(1-R_{x}\right) G H_{g s}+G\left(H_{0}-H_{i}\right)=G H_{0}-R_{x} G H_{s s} \\
& E_{2}=\left(\dot{m}_{v I}+\dot{m}_{v 2}\right)\left(H_{g s}-H_{s s}-H_{g s}\right)=-\left(\dot{m}_{v 1}+\dot{m}_{v 2}\right) H_{s s}
\end{aligned}
$$

and

$$
E_{3}=\sum\left(-\dot{m}_{i}\left(\left.H_{m}\right|_{m_{t} \geq 0} \text { or }\left.H_{a}\right|_{m_{i}<0}\right)\right)+E_{c}
$$

where
$\mathrm{H}_{\mathrm{gg}}$ is the enthalpy of $\mathrm{CO}_{2}$ gas at its saturation temperature,
$\mathrm{H}_{\mathrm{ss}}$ is the enthalpy of solid $\mathrm{CO}_{2}$ at its saturation temperature,
$\mathrm{H}_{0}$ is the enthalpy of pressurized $\mathrm{CO}_{2}$ at the nozzle,
$\mathrm{H}_{\mathrm{m}}$ is the enthalpy of the $\mathrm{CO}_{2}$-air mixture,
$\mathrm{H}_{\mathrm{a}}$ is the enthalpy of dry ambient air.
$E_{c}$ is the enthalpy input of water vapor in the ambient air entering the enclosure.

Heat transfer through the enclosure walls/floor/ceiling during the discharge period can be calculated by using the thermally thick approximate solution of the heat conduction equation with a convective heat transfer boundary conditon [3]. However, many applications demand a thermally intermediate solution for the extended period following discharge. In anticipation of the need for a solution that 
will describe the heat transfer during the entire discharge test, the transient, one-dimensional heat conduction equation is solved using a finite difference procedure [3] when the wall thickness is less than $2(\alpha t)^{0.5}$, where $t$ now is the total duration of the simulated test. The enclosure wall heat transfer using the finite difference solution and an assumed solid $\mathrm{CO}_{2}$ floor layer accumulating linearly with time, can be written as

$$
E_{4}=-h_{f} F_{1}\left[\left(A_{d}-A \frac{t}{t_{d}}\right)\left(T-T_{w, 1}\right)+A \frac{t}{t_{d}}\left(T_{s a t}-T_{w, 1}\right)\right]
$$

where

$T_{w, l}$ is the inner wall surface temperature at time $t$,

$h_{i}$ is the convective heat transfer coefficient at the inner wall of the enclosure,

$A_{3}$ is the total internal surface area of the enclosure, $t_{d}$ is the duration of $\mathrm{CO}_{2}$ discharge.

$\mathrm{T}_{\text {sat }}$ is the saturation temperature of solid $\mathrm{CO}_{2}$ accumulating on the floor,

and $\quad F_{1}$ is a user supplied correction factor to account for heterogeneous enclosure surfaces containing windows, doors, protusions, etc that may act as heat sources or sinks.

The inner wall convective heat transfer coefficient used in Eqn 13 is evaluated using a turbulent flow correlation [3] where we assume the effective velocity at the inner wall is a combination of the velocities induced by discharge and by leakage flow. The discharge flow induced velocity is assumed to be twice the $\mathrm{CO}_{2}$ particle terminal velocity, and the leakage flow characteristic velocity is an area weighted fraction of the maximum velocity through the leakage openings.

Once $E_{1}$ through $E_{4}$ and the specific heats are evaluated, the energy conservation equation is

$$
\left(\rho V C_{v}+W V C_{p w w}+V H_{w} \frac{d W}{d T}\right) \frac{d T}{d t}=E 1-E 2+E 3-E 4-V U \frac{d \rho}{d t}-E_{w} \frac{d T_{w}}{d t}
$$

where $\mathrm{C}_{\mathrm{v}}$ is the constant volume specific heat of the $\mathrm{CO}_{2}$ - air mixture, and

$$
E_{w}=I\left[\left(W-W_{s}-W_{s s}\right) C_{p w i}+W_{s s} C_{p w s}\right]
$$

The ideal gas law equation as applied to this mixture of $\mathrm{CO}_{2}$ and air is

$$
P=\frac{\rho R T}{\bar{M}}=\frac{\rho R T}{(1-x) M_{a}+x M_{c}}=\rho R T\left(\frac{1-y_{m}}{M_{c}}+\frac{y_{m}}{M_{c}}\right)
$$

where

$\mathbf{P}$ is the pressure at the enclosure bottom,

and $\mathbf{R}$ is the universal gas constant.

Differentiating both sides of Eqn 16 yields 


$$
\frac{d P}{d t}=\left(\frac{1-y_{m}}{M_{a}}+\frac{y_{m}}{M_{c}}\right) R\left(\rho \frac{d T}{d t}+T \frac{d \rho}{d t}\right)+\rho R T\left(\frac{1}{M_{c}}-\frac{1}{M_{a}}\right) \frac{d y_{m}}{d t}
$$

The total rate of pressure change consists of Eqn 17 plus the contribution of the water vapor pressure, $P_{\text {vap }}$, to give as follows

$$
\frac{d P}{d t}=\frac{-P\left(M_{c}-M_{a}\right) \frac{d x}{d t}+R T \frac{d p}{d t}+R \rho \frac{d T}{d t}}{(1-x) M_{a}+x M_{c}}+\left(W R+R T \frac{d W_{2}}{d T}\right) \frac{d T}{d t}
$$

Equations 1,8,14, and 18, plus the auxiliary equations above, represent a set of four first order ordinary differential equations in the dependent variables $\rho, x$ or $y_{m}, T$ and $P$. Solution techniques are described after presenting the formulation for the post-discharge model.

\section{Post-Discharge Model}

After discharge is completed, there is no source of turbulent mixing to maintain uniform temperatures and $\mathrm{CO}_{2}$ concentrations in the enclosure. As the turbulence level decays in the few seconds following discharge (or, more precisely, in the final stage of discharge in which only $\mathrm{CO}_{2}$ vapor is discharged), air leakage in through openings in the upper portion of the enclosure, and cold $\mathrm{CO}_{2}$-air mixture outleakage through openings in the lower portion of the enclosure create stratified temperatures and concentrations. This transition period in which the enclosure evolves from a uniform to a stratified environment is modeled with the same equations as during discharge, but with $\mathrm{G}$ set equal to zero, with a linearly decreasing average enclosure velocity for heat/mass transfer calculations, and with density, temperature, and concentration now representing average values as nonuniformities develop.

The post-discharge model, which is invoked after a transition period equal to the discharge time, assumes that both the temperature and mixture density are linearly stratified as illustrated in Figure

2. The equations representing the assumed distributions are

$$
\rho=\frac{\rho_{h}-\rho_{0}}{h} z+\rho_{0}
$$

and

$$
T=\frac{T_{h}-T_{o}}{h} z+T_{0}
$$

where $\rho_{h}$ and $\rho_{0}$ are mixture densities at the top and bottom of the enclosure, respectively, and $T_{h}$ and $\mathrm{T}_{0}$ are the temperatures at the top and bottom of the enclosure.

Hydrostatic pressure distributions and ideal gas law equations are employed to relate the linear density and temperature distributions to the corresponding nonlinear pressure and $\mathrm{CO}_{2}$ concentration distributions shown in Figure 2. 
Conservation of mass for this assumed distribution of gas density in the enclosure is

$$
\frac{V}{2} \frac{d\left(p_{h}+\rho_{0}\right)}{d t}=-\sum \dot{m}_{1}+\dot{m}_{v 2}
$$

Leakage mass flow rates are calculated from Equations 2 and 3 as in the discharge model, but the enclosure pressure at elevation $b_{i}$ of the ith leakage area is now a quadratic function of elevation. The evaporation rate from the shrinking carbon dioxide floor layer, denoted by the last term in Eqn 23, is calculated from the mass fraction of carbon dioxide at floor level and a mass transfer coefficient based on the lowest elevation leakage velocity. Details are given in [3].

$$
\dot{m}_{d c}=D_{f} A\left[\frac{M_{c}}{M_{c}-M_{a}} \frac{\rho_{0}-P_{h}}{h}+\frac{M_{d} M_{c} P}{\left(M_{c}-M_{a}\right) R} \frac{\frac{T_{0}-T_{h}}{h}}{\left(\frac{T_{0}+T_{h}}{2}\right)^{2}}\right]
$$

and

$$
\dot{m}_{d a}=D_{f} A\left[\frac{M_{a}}{M_{c}-M_{a}} \frac{\rho_{0}-P_{h}}{h}+\frac{M_{d} M_{c} P}{\left(M_{c}-M_{a}\right) R} \frac{\frac{T_{0}-T_{h}}{h}}{\left(\frac{T_{0}+T_{h}}{2}\right)^{2}}\right]
$$

Conservation of carbon dioxide in the post-discharge model is given by

$$
\frac{d}{d t} \int_{0}^{h} \rho y_{m} A d z=m_{v 2}-y_{m} \sum m_{i}
$$

Conservation of energy in the post-discharge model is expressed as

$$
\frac{d}{d t} \int_{0}^{h} \rho U A d z=E_{3}-\dot{m}_{2} H_{s z}+E_{7}-E_{w}
$$

where the left hand side represents the rate of increase of mixture internal energy, $\mathrm{E}_{\mathbf{3}}$ is the net rate of energy input (or outflow) from air (or $\mathrm{CO}_{2}$ ), $\mathrm{E}_{7}$ is the rate of energy input by natural convection from the walls, and $\mathrm{E}_{\mathrm{w}}$ is the rate of energy absorbed by water or ice suspended in the gas mixture.

Additional equations are needed to determine the temperature and density gradients in the enclosure following discharge. These equations are obtained by considering the conservation equations for the upper half of the enclosure. For example, conservation of mass in the upper half volume of the enclosure can be written as 


$$
\begin{aligned}
\frac{V d\left(\frac{\rho_{h / 2}+\rho_{h}}{2}\right)}{2} & =\frac{V}{2}\left(\frac{3}{4} \frac{d \rho_{h}}{d t}+\frac{1}{4} \frac{d \rho_{o}}{d t}\right) \\
& =-\left.\left(\sum \dot{m}_{i}\right)\right|_{b_{t}>\frac{h}{2}}-\dot{m}_{d a}+\dot{m}_{d c}-\dot{m}_{t}
\end{aligned}
$$

where the first term on the right hand side represents the net air leakage above the enclosure midplane, the second term represents the diffusion of air from the upper volume to the lower volume, the third term represents the diffusion of $\mathrm{CO}_{2}$ from the lower volume to the upper volume, and the last term denotes the mass transfer between the upper and lower volumes. The diffusion terms can be expressed as follows after evaluating the air and $\mathrm{CO}_{2}$ concentration gradients at the midplane.

$$
\begin{gathered}
\dot{m}_{d c}=D_{f} A\left[\frac{M_{c}}{M_{c}-M_{a}} \frac{\rho_{0}-\rho_{h}}{h}+\frac{M_{a} M_{c} P}{\left(M_{c}-M_{a}\right) R} \frac{\frac{T_{0}-T_{h}}{h}}{\left(\frac{T_{0}+T_{h}}{2}\right)^{2}}\right] \\
\dot{m}_{d a}=D_{f} A\left[\frac{M_{a}}{M_{c}-M_{e}} \frac{\rho_{0}-\rho_{h}}{h}+\frac{M_{d} M_{c} P}{\left(M_{c}-M_{a}\right) R} \frac{\frac{T_{0}-T_{h}}{h}}{\left(\frac{T_{0}+T_{h}}{2}\right)^{2}}\right]
\end{gathered}
$$

Conservation of $\mathrm{CO}_{2}$ in the upper volume is similar to Eqn 24 except that the integration and summation start at the enclosure midplane. Conservation of energy in the upper volume is similar to Eqns 14 and 25 except that there is no floor evaporation term and the heat transfer through the upper walls/ceiling (denoted by $\mathrm{E}_{10}$ below) is assumed equal to half the heat transfer for the entire surface area.

Equations $21,24,25$, and the three conservation equations for the upper volume can be written as a set of 6 differential equations for the unknowns $P, \rho_{o}, \rho_{h}, m_{b} T_{0}$, and $T_{h}$ subject to initial conditions at $\mathrm{t}=\mathrm{t}_{\mathrm{cd}}$.

\section{Numerical Solution Method}

The numerical method used to obtain solutions for the discharge model and the post-discharge model is the Runge-Kutta Fehlberg method with time step determined according to a user specified allowable error for each dependent variable [4]. In the transition period immediately following discharge, the set of simultaneous algebraic equations is solved using the Gauss-Jordan Elimination method. Both methods were implemented via a Fortran program using published algorithms [5]. The computer program was compiled and executed on the WPI DEC workstations and on a $386 \mathrm{PC}$. 


\section{CALCULATED RESULTS COMPARED TO TEST DATA}

Model calculations have been compared to data obtained in a series of $\mathrm{CO}_{2}$ discharge tests conducted at Factory Mutual Research Corporation for the Pennsylvania Power \& Light Company [6]. The tests were conducted in a $13.4 \mathrm{~m} \times 12.2 \mathrm{~m} \times 6.1 \mathrm{~m}$ high enclosure with walls and ceiling constructed of 1 inch $(2.54 \mathrm{~cm})$ thick Marinite I panels. Access doors and windows were provided on two walls, and a series of holes was drilled in two walls to avoid pressure damage to the structure. The total leakage area (including gaps around doors, panels, etc.) was determined by door fan pressurization tests to be $0.192 \mathrm{~m}^{2}\left(297 \mathrm{in}^{2}\right)$, whereas the area of the drilled holes was $0.138 \mathrm{~m}^{2}\left(214 \mathrm{in}^{2}\right)$.

Different amounts of $\mathrm{CO}_{2}$ were released in the three tests conducted. The amounts and discharge times for Tests 2 and 3 , as measured by load cells under the $\mathrm{CO}_{2}$ tank were: Test 2: $1255 \mathrm{~kg}$ of $\mathrm{CO}_{2}$ discharged over $207 \mathrm{sec}$; Test 3: $1709 \mathrm{~kg}$ of $\mathrm{CO}_{2}$ discharged over $281 \mathrm{sec}$. Based on the NFPA 12 [1] flooding factors, $1327 \mathrm{~kg}$ of $\mathrm{CO}_{2}$ should have been adequate to generate a $\mathrm{CO}_{2}$ concentration of $50 \mathrm{v} \%$ in the $993 \mathrm{~m}^{3}$ enclosure. Thus the expected peak concentrations (implied by NFPA 12 for the actual discharged quantities of $\mathrm{CO}_{2}$ ) would have been about $47 \mathrm{v} \%$ for Test 2 and about $64 \mathrm{v} \%$ for Test 3.

Measurements at several elevations included temperatures of the gas and the enclosure walls, $\mathrm{CO}_{2}$ gas concentrations (measured with both infrared analyzers and thermal conductivity analyzers), and differential pressures at two elevations across one wall.

Enclosure gas phase temperatures and pressures in Test 2 are plotted in Figures 3 and 4, respectively. The temperatures rapidly drop from ambient to the one atmosphere saturation temperature of $\mathrm{CO}_{2}$ $\left(194^{\circ} \mathrm{K}\right)$ about two minutes after discharge begins. It remains saturated for several minutes following discharge, and then gradually warms. The calculated temperatures track the measurements during the cooldown, but somewhat underestimate the rate of warming. This may be due to the additional heat transfer surface area associated with the structural members and instrumentation. Increasing the enclosure surface area by $10 \%$, to represent these surfaces provided better agreement with the temperature data [3].

The pressure in Figure 4 first drops as the enclosure air is cooled by the cold $\mathrm{CO}_{2}$ discharged, and then increases to a maximum as additional moles of gaseous $\mathrm{CO}_{2}$ are discharged and evaporated. The calculated maximum pressure rise of about $200 \mathrm{~Pa}$ is very close to the measured value. The calculated peak occurs about one minute prior to the completion of discharge while the measured peak occurs 30-40 sec after the end of discharge. The differences may be due to transients and phase changes in the $\mathrm{CO}_{2}$ delivery piping. The calculations assume a constant flow rate of liquid $\mathrm{CO}_{2}$, but the actual discharge probably consists of primarily gaseous $\mathrm{CO}_{2}$ before the pipe walls cool, and after the liquid is discharged from the tank but the pipe/tank still contains pressurized gaseous $\mathrm{CO}_{2}$. The sharper decay after the calculated peak is probably also due to this effect. A less significant error source is the assumed solid $\mathrm{CO}_{2}$ particle diameter $(0.3 \mathrm{~mm})$ which may too small and therefore lead to an overestimate of $\mathrm{CO}_{2}$ vaporization rates. Calculated and measured pressures gradually return to the pre-discharge value as the enclosure gas warms.

Calculated and measured $\mathrm{CO}_{2}$ concentration histories for Test 2 are shown in Figure 5. The maximum calculated concentration of $40 \mathrm{v} \%$ agrees with the data and occurs a few minutes after 
discharge is completed. The time lag is due to the time required to evaporate the remaining dry ice on the enclosure floor and suspended in air. After the maximum concentration is reached, the concentration falls more rapidly at the upper elevations than the lower elevations of the enclosure. Calculated and measured concentration histories at all elevations agree for the approximately 35 minute test duration.

The calculated and measured $\mathrm{CO}_{2}$ concentrations in Test 3 are shown in Figure 6 . There is excellent agreement. The $50 \mathrm{v} \%$ peak concentration in Test 3 is about $10 \mathrm{v} \%$ higher than in Test 2, and requires considerably more $\mathrm{CO}_{2}$ to be discharged than the NFPA 12 flooding factors require. The calculated rates of concentration decay at all four elevations are almost identical to the measurements. Thus, the assumed concentration distribution and the calculated leakage rates appear to be verified, at least for this test series.

Several parameter sensitivity calculations were conducted with the model simulations of these discharge test conditions. Among the many interesting results of these caclulations was the observation that varying the solid $\mathrm{CO}_{2}$ evaporation rate (via heat transfer coefficient or particle size) during discharge did not significantly affect the peak concentration (because it was compensated by leakage from the enclosure while the pressure was high) but that additional heat transfer after discharge could increase the peak $\mathrm{CO}_{2}$ concentration. This suggests that performing $\mathrm{CO}_{2}$ discharge test on a warm day is more likely to produce success than conducting the same test on a cold day.

\section{REFERENCES}

1. NFPA 12, "Carbon Dioxide Extinguishing Systems," National Fire Protection Association, 1993.

2. DiNenno, P.J., Forssell, E.W., Starchville, M.D., and Carhart, H.W., "Evaluation of Halon 1301 Test Gas Simulants: Enclosure Leakage," Fire Technology, v 24, pp 24-40, 1989.

3. Wai Hung, Cheng "Modelling of Carbon Dioxide Total Flooding Discharge Tests," M.S. Thesis, Worcester Polytechnic Institute, July 1991.

4. Mathews, J.H., Numerical Methods for Computer Science, Engineering and Mathematics, Prentice Hall, 1987.

5. Press, W.H., Flannery, S.A., Teukolsky, S.A., and Vetterling, W.T., Numerical Recipes, The Art of Computing (Fortran Version), Cambridge University Press, 1990.

6. Kohn D.J., "Alternative to $\mathrm{CO}_{2}$ Full Discharge Testing, Susquehanna Steam Electric Station" PP\&L Report, March 1990.

7. NFPA 12A, "Standard on Halon 1301 Fire Extinguishing Systems," National Fire Protection Association, 1992. 
Figure 1 Model for During Discharge
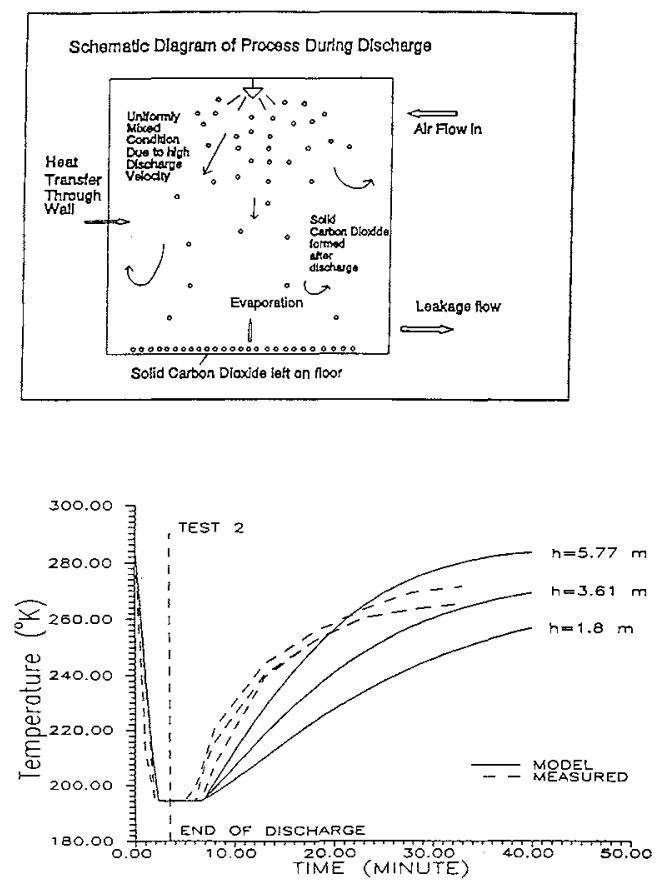

Figure 3 Temperature from Hodel vs rest 2

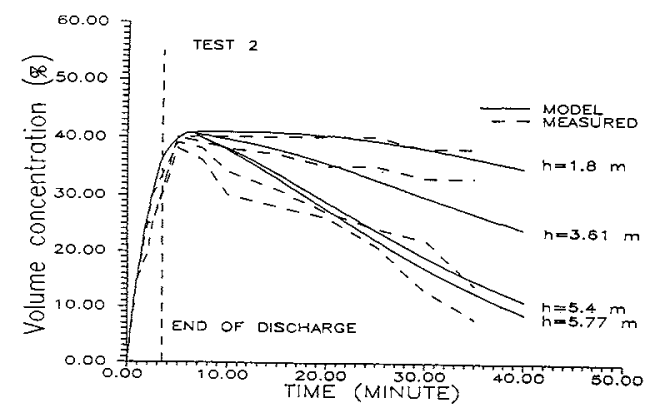

Figure 5 concentration from Hodel vo Test 2
Figure 2 Model for After Discharge
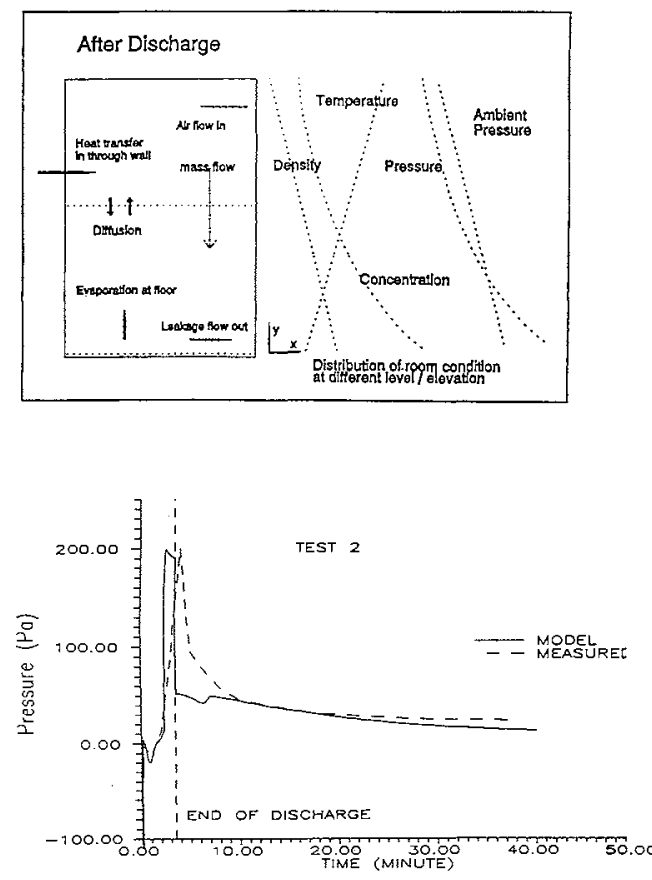

Figure 4 Pressure from hodel vs Test 2

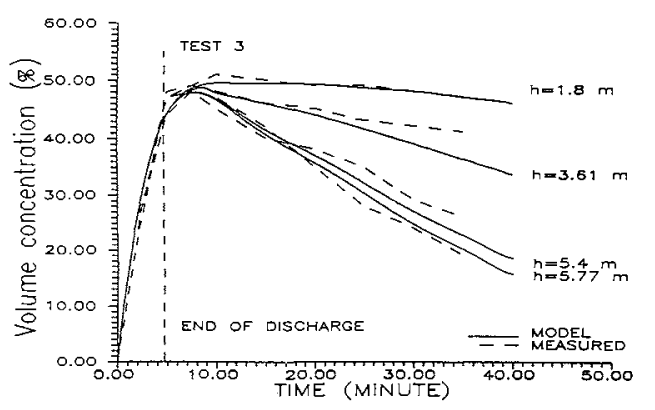

Figure 6 Concentration from Model vs Test 3 